\title{
Resonance shift effects in apertureless scanning near-field optical microscopy
}

\author{
J. A. Porto, ${ }^{1}$ P. Johansson, ${ }^{2}$ S. P. Apell, ${ }^{1}$ and T. López-Rios ${ }^{3}$ \\ ${ }^{1}$ Department of Applied Physics, Chalmers University of Technology and Göteborg University, S-41296 Göteborg, Sweden \\ ${ }^{2}$ Department of Natural Sciences, University of Örebro, S-701 82 Örebro, Sweden \\ ${ }^{3}$ Laboratoire d'Etudes des Propriétés Electroniques des Solides, \\ (LEPES/CNRS), BP 166, 38042 Grenoble Cedex 9, France
}

(Dated: October 25, 2018)

\begin{abstract}
We develop a theory to study apertureless scanning near-field optical microscopy which takes into account retardation, higher multipoles of the tip, and the multiple scattering between the tip and the surface. We focus on metallic systems and discuss the implication of the formation of tip-induced surface plasmon modes in the tip-surface system. We discuss the effects associated with the shift in energy of those modes as a function of the tip-surface distance. Both the local field and the scattering cross section are enhanced when the tip approaches the surface, but there is no general correspondence between the two enhancements.
\end{abstract}

PACS numbers: 68.37.Uv,73.22.Lp,78.68.+m

\section{INTRODUCTION}

Scanning near-field optical microscopy (SNOM) 国目 is a technique that allows to obtain optical images of objects with subpravelength resolution. In the pioneering experiments, 1 : subwavelength optical apertures where used to illuminate the sample and obtain subwavelength images. Nevertheless, it is also possible to perform nearfield optical imaging without case of apertureless SNOM, Al 6 . 18 which has attracted considerable attention in recent years, allowing interesting experiments such as near-field fluorescence imaging 9 and near-field vibrational absorption detection. $10 \mathrm{In}$ apertureless SNOM, light is focused at the tip of a scanning probe microscope (SPM) and the field enhancement near the tip is used to obtain the subwavelength imaging. Apertureless SNOM allows to perform simultaneously near-field optical imaging and other types of local probe microscopies, such as atomic force microscopy (AFM) or scanning tunneling microscopy (STM). In addition, it has provided higher resolution than other SNOM techniques. A key issue in the apertureless SNOM technique is the tip-sample coupling, and how the electromagnetic resonances at the cavity formed by the tip and the surface may affect the corresponding scattered field.

There has been an extensive theoretical research about SNOM, 11 - 2 mostly related to imaging with aperture probes. With regard to apertureless SNOM, it has been studied theoretically considering different models and approximations such as electric dipole for the tip 13,4 the electrostatic approximation 15 or the passive probe approximation.16 It has also been studied in two dimensions.17 There have been detailed numerical calculations of the near field associated with apertureless probes $18,12,20$ and the field enhancement has been discussed in the context of apermueless SNOM.21 In addition, the role of tip geometry,22.23.24 the influence of tip modulation 25 and the implications for magneto-optical near-field imaginge6 27 have been studied. Nevertheless, there is a need for clarification of how the tip-sample coupling affects the signal detected and the local-field enhancement. In particular, the question concerning the link between electromagnetic resonances at the cavity formed by the tip and the surface and the corresponding scattered field needs to be addressed. These resonances concern free electron metals for which surface plasmons may exist. In the simple case of an isolated particle it is well known that the scattering and extinction cross sections have resonance maxima at about the same photon energy. This is obvious from the fact that large charges at the surface give rise to large dipoles and consequently large radiation. If we instead consider two interacting particles the situation is not so simple. In this case there is not a direct_correlation between near-field and far-field resonances.28.29

This problem is of the highest relevance concerning the emerging field of optical spectroscopy with an apertureless SNOM, which comprises areas such as near-field Raman scattering and luminescence. The small number of molecules between the tip and the surface leads to extremely small signals that are very difficult to measure. One possible way to overcome this problem is to take advantage of the resonance that may exist at the cavity between the tip and the surface and the extremely high fields associated.30 In this context, a key question is what is the signature of this type of resonances on the far field and on the optical images.

In this article we develop a theory which allows to perform a detailed study of the tip-sample interaction in apertureless SNOM. We model the tip by a sphere of finite size, whose radius may correspond roughly to the radius of curvature of the real tip. The main features of the theory are: (i) retardation, (ii) higher multipoles of the sphere, and (iii) the multiple scattering between the sphere and the surface are taken into account 26 We use this theory to analyze the effects associated with the shift in energy of the surface-plasmon resonances of the tipsample system as the tip approaches the surface. Those resonances produce an enhancement in the near field and in the far field as well, although there is not always an ex- 
act correspondence between the two enhancements. We will also discuss the convergence of the results with the number of multipoles taken into account in the calculation. As a general result, a high number of multipoles should be retained to arrive at convergence.

The paper is organized as follows. In Sec. II we highlight the main points of the theoretical framework used for the calculations. In Sec. III we discuss the numerical results obtained for the near-field enhancement and scattering cross sections as a function of different parameters of the system. In Sec. IV, we present the concluding remarks.

\section{METHOD}

In this section we present the main features of the theoretical formalism employed.26 Figure 11(a) shows the system under study. A flat metallic sample, filling the half-space $z<z_{0}=-(R+d)$, is probed by a metallic tip, modeled by a sphere with radius $R$. The distance between the sphere and the surface is $d$, as shown in Fig. 11(a).

We are interested in studying how this system scatters the incident light and which local electromagnetic fields are built up in the process. For that purpose, we consider a plane wave incident on this system with wave vector $\mathbf{q}$, which can be written as (an $e^{-i \omega t}$ factor is omitted in fields and other quantities in what follows)

$$
\mathbf{E}_{i n}(\mathbf{r})=\left\{E^{(s)} \hat{\mathbf{s}}+E^{(p)} \hat{\mathbf{p}}\right\} e^{i \mathbf{q} \cdot \mathbf{r}} .
$$

For a wave vector $\mathbf{q}$, the polarization vectors for $\mathrm{s}$ and $\mathrm{p}$ polarization are, respectively, $\hat{\mathbf{s}}=\hat{\mathbf{z}} \times \hat{\mathbf{q}}_{\|}$, and $\hat{\mathbf{p}}=$ $-\hat{\mathbf{q}} \times\left(\hat{\mathbf{z}} \times \hat{\mathbf{q}}_{\|}\right)$, where $\mathbf{q}_{\|}$is the projection of $\mathbf{q}$ in the surface plane. In the present work we will mainly focus on the p-polarization.

In order to tackle the scattering off the sphere-surface system, the electromagnetic field inside and just outside the sphere is expanded in terms of the electric (E) and magnetic (M) multipoles. 31 The electric field inside the sphere can be expressed as

$$
\mathbf{E}=\sum_{l m} k c_{l m}^{(M)} j_{l}\left(k_{T} r\right) \mathbf{X}_{l m}+\frac{i}{\epsilon_{T}} \nabla \times\left[c_{l m}^{(E)} j_{l}\left(k_{T} r\right) \mathbf{X}_{l m}\right]
$$

The electric field just outside the sphere can be written as the linear combination of incident and outgoing waves from the sphere

$$
\begin{aligned}
\mathbf{E}= & \sum_{l m} k\left[a_{l m}^{(M)} j_{l}(k r)+b_{l m}^{(M)} h_{l}(k r)\right] \mathbf{X}_{l m}+ \\
& +i \nabla \times\left\{\left[a_{l m}^{(E)} j_{l}(k r)+b_{l m}^{(E)} h_{l}(k r)\right] \mathbf{X}_{l m}\right\}
\end{aligned}
$$

where $\mathbf{X}_{l m}$ is the vector spherical harmonics, $j_{l}$ denotes the spherical Bessel functions related to incoming waves, and $h_{l}$ are the spherical Hankel functions related to outgoing waves. Equivalent expressions can be written for the magnetic field. (a)

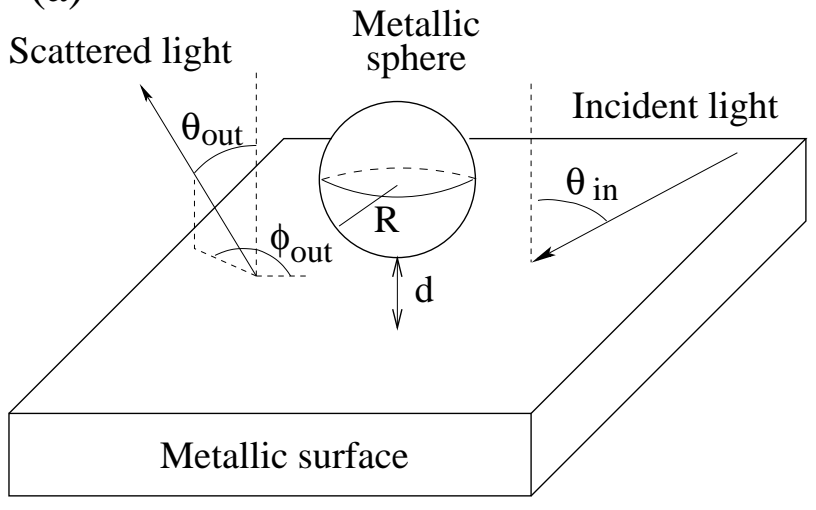

(b)

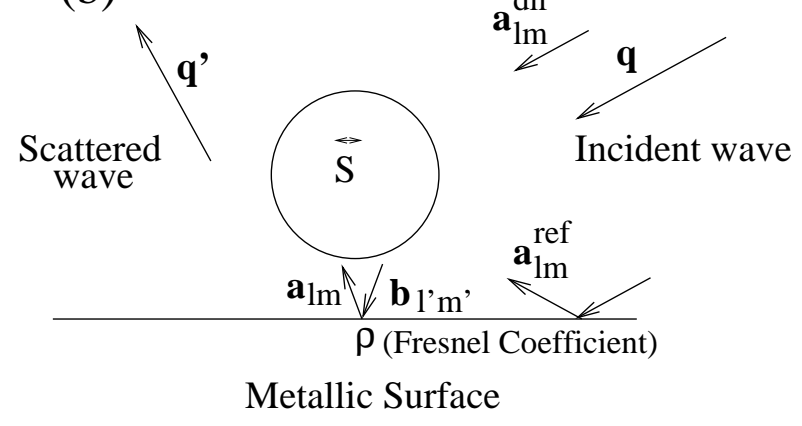

FIG. 1: (a) Model geometry used in the calculation and (b) illustration of the scheme used in the calculation

By imposing the continuity of the tangential components of $\mathbf{E}$ and $\mathbf{H}$ and the normal components of $\mathbf{B}$ and $\mathbf{D}$ at the sphere surface, it is possible to relate the coefficient of the outgoing wave to the incoming wave for each electric and magnetic multiple:

$$
s_{l}^{(E)}=\frac{b_{l m}^{(E)}}{a_{l m}^{(E)}}, \text { and } s_{l}^{(M)}=\frac{b_{l m}^{(M)}}{a_{l m}^{(M)}},
$$

where $s_{l}^{(E)}$ and $s_{l}^{(M)}$ are sphere response functions. Due to the symmetry of the sphere, the "quantum" number $l$ is conserved in a scattering event and the response functions are independent of $m$. These expressions can be rewritten in a matrix form

$$
\vec{b}=\overleftrightarrow{s} \vec{a}
$$

where $\overleftrightarrow{s}$ is a diagonal tensor that relates the incoming wave coefficients, $\vec{a}$, to the outgoing waves, $\vec{b}$.

In the situation under study, the field impinging on the sphere consists of three contributions [see Fig. 11(b)],

$$
\vec{a}=\vec{a}^{\mathrm{dir}}+\vec{a}^{\mathrm{ref}}+\overleftrightarrow{N} \vec{a}
$$

$\vec{a}^{\text {dir }}$ corresponds to the amplitude of the field from the original incident wave, its value can be obtained by expanding the incident plane wave in the multipoles of the 
sphere, while $\vec{a}^{\text {ref }}$ represents the amplitude of the incident wave reflected once from the sample surface. Its value is obtained by expanding the reflected plane wave from the surface in the multipoles of the sphere. Finally, $\overleftrightarrow{N} \vec{a}$ represents waves scattered from the sphere that return to it after reflection from the sample. This term contains the exact field described by $\vec{a}$, therefore it accounts for all multiple scattering events in which waves are scattered between the sphere and the sample an arbitrary number of times. $\overleftrightarrow{N}$ is a tensor in multipole space. It indicates how a spherical wave with a given angular momenta 1 and $m$ is reflected from the surface and returns to the sphere. In this reflection process the quantum number $l$ is not necessarily conserved but, thanks to the cylindrical symmetry, $m$ is. In the coupling between the sphere and the sample, both propagating and evanescent waves are taken into account.

Retaining a finite number of multipoles, Eq. ([D) can be solved by a matrix inversion

$$
\vec{a}=[\overleftrightarrow{1}-\overleftrightarrow{N}]^{-1}\left(\vec{a}^{\mathrm{dir}}+\vec{a}^{\mathrm{ref}}\right)
$$

Now, from Eq. (何) we can obtain the coefficients for the outgoing waves from the sphere. From those coefficients it is possible to calculate the radiated field from the sphere-surface system in a particular direction given by the wave vector $\mathbf{q}^{\prime}$,

$$
\mathbf{E}_{\mathrm{rad}}=\left\{E_{\mathrm{rad}}^{(s)} \hat{\mathbf{s}}^{\prime}+E_{\mathrm{rad}}^{(p)} \hat{\mathbf{p}}^{\prime}\right\} e^{i \mathbf{q}^{\prime} \cdot \mathbf{r}}
$$

If we consider a direction other than the specular direction, the radiated field comprises two contributions:

$$
\mathbf{E}_{\mathrm{rad}}=\mathbf{E}_{\mathrm{rad}, \text { sphere }}+\mathbf{E}_{\mathrm{rad}, \text { surface }},
$$

where $\mathbf{E}_{\mathrm{rad} \text {,sphere }}$ represents the field radiated directly by the sphere, and $\mathbf{E}_{\text {rad,surface }}$ corresponds to waves having a final scattering event from the surface.

\section{RESULTS AND DISCUSSION}

In this section, we study the system formed by a gold sphere and a gold surface by means of the theoretical method described above. The dielectric function of gold is described using the tables reported in Ref. 32. We will consider an incident p-polarized wave with angle of incidence $\theta_{i n}=57^{\circ}$. We will mainly concentrate on the effects associated with the formation of resonances of the tip-surface coupled system. In what follows, we will mainly focus on the analysis of the local field enhancement and the scattering cross section for scattered p-polarized light. Results will be shown for the observation angles $\theta_{\text {out }}=57^{\circ}$ and $\phi_{\text {out }}=90^{\circ}$. It should be noticed that similar effects than the ones discussed in the present work can be seen for other angles of observation and incidence.
Figure 2(a) shows the local-field enhancement below the sphere as a function of energy for different values of the tip-sample distance and a sphere radius of $40 \mathrm{~nm}$. The peak that is built up as the sphere approaches the surface is due to a surface plasmon of the coupled system formed by the sphere and the surface. 30 This maximum experiences a redshift as the tip approaches the surface. The scattered light from the system shows a similar peak, as illustrated by Fig. 2(b).

Figure 3(a) shows the local-field enhancement when a larger sphere (radius of $80 \mathrm{~nm}$ ) is considered. The first peak has moved to lower energy (compared to the previous case with smaller radius) and a new peak appears at
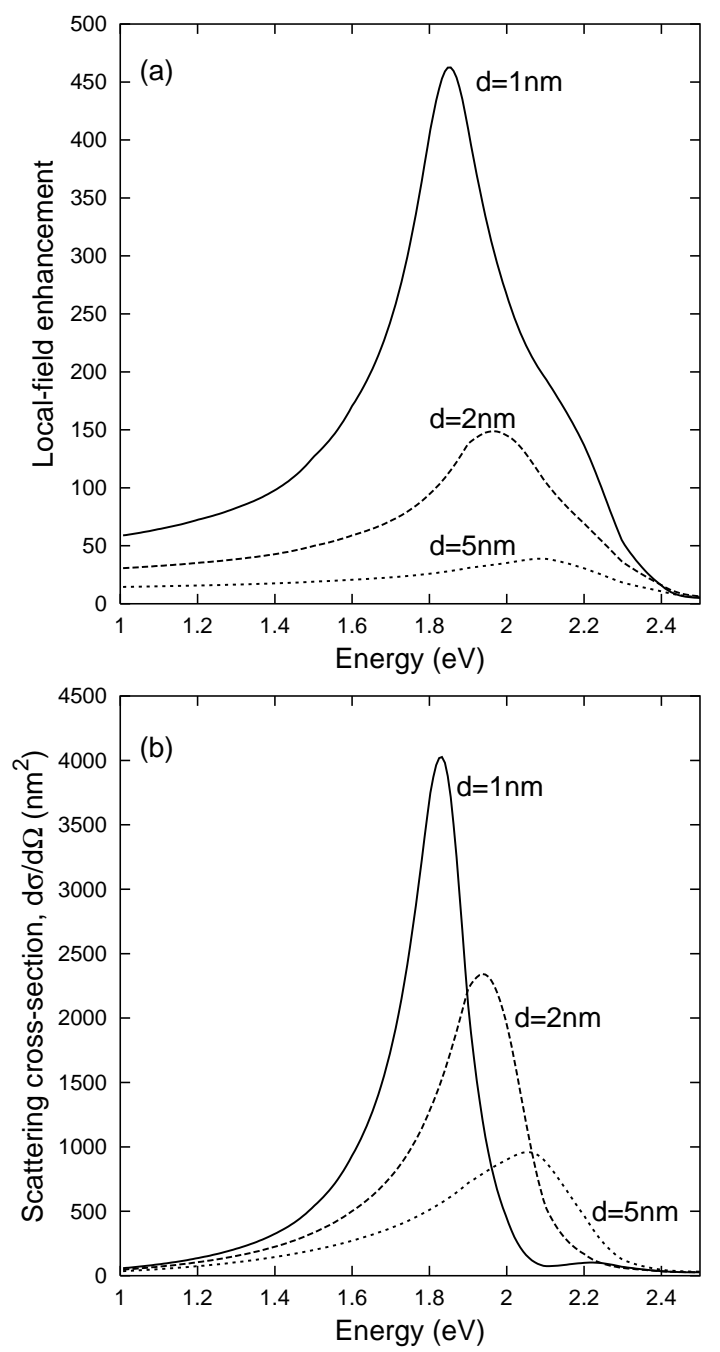

FIG. 2: (a) Near-field enhancement just underneath the sphere and (b) differential scattering cross section as a function of the energy of the incident wave for a gold sphere of radius $\mathrm{R}=40 \mathrm{~nm}$, and for different values of the distance between the tip and the surface, $d$. The angle of incidence is $\theta_{\text {in }}=57^{\circ}$ and the angles of observation are $\theta_{\text {out }}=57^{\circ}$ and $\phi_{\text {out }}=90^{\circ}$. 
higher energy, but the field enhancement does not vary substantially over a wide range of energies. The scattering cross section has a qualitatively different behavior [see Fig. 3(b)]. The first peak appears also in the scattering cross section. By contrast, for higher energies, there is no significant correspondence between the scattering cross section and the local field enhancement.

In order to analyze the different features in Figs. 2 and 3, we study the distribution of the electric field near the sphere. Figure 1 represents the electric field around the $80 \mathrm{~nm}$-radius sphere for the first field enhancement maximum in Fig. 3(a). This figure, as well as other vector plots shown below, displays the electric
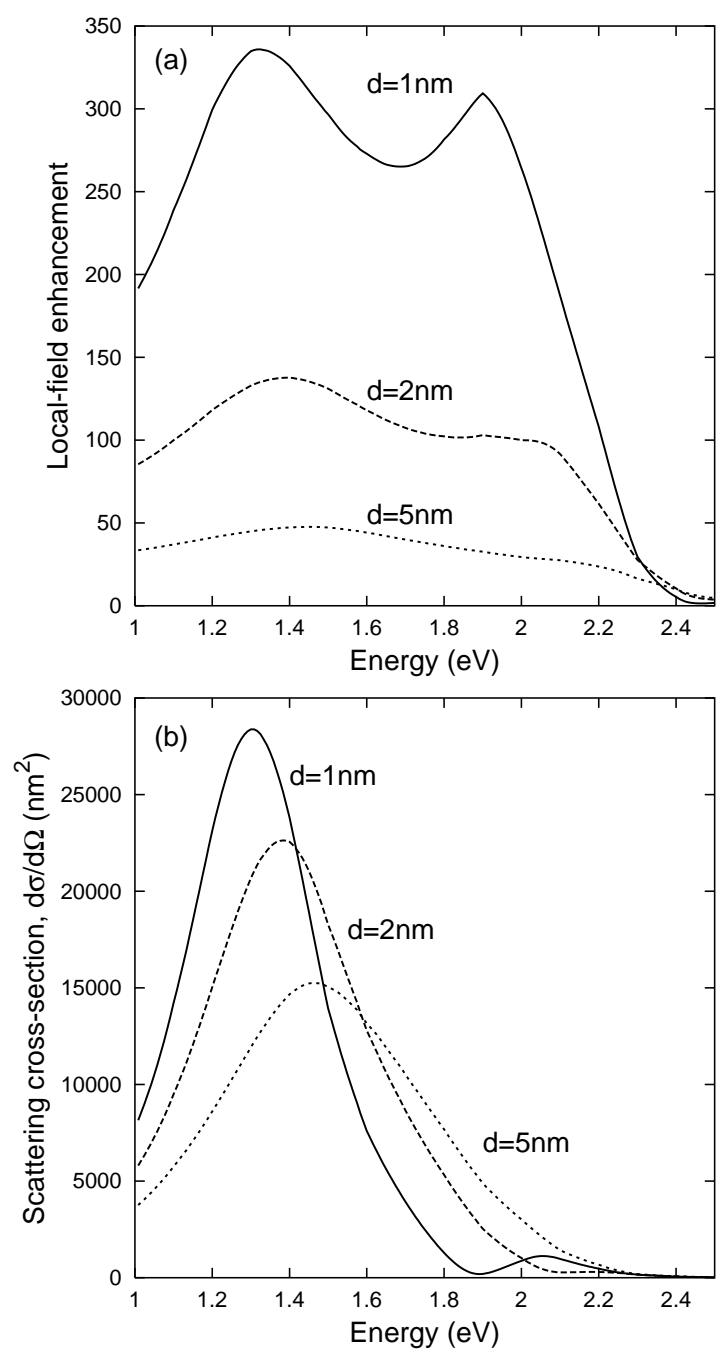

FIG. 3: (a) Near-field enhancement just underneath the sphere and (b) differential scattering cross section as a function of the energy of the incident wave for a gold sphere of radius $R=80 \mathrm{~nm}$, and for different values of the distance between the tip and the surface, $d$. The angle of incidence is $\theta_{\text {in }}=57^{\circ}$ and the angles of observation are $\theta_{\text {out }}=57^{\circ}$ and $\phi_{\text {out }}=90^{\circ}$. field just outside the sphere in a vertical plane that also contains the incident wave vector $\mathbf{q}$. The fields shown in Fig. 14 corresponds to the fundamental resonant surfaceplasmon mode of the tip-surface system. This fundamental mode is also responsible for the peak in Fig. 2(a), where a smaller radius is considered. The local field in that case shows a very similar pattern. However, for the second peak in Fig 3(a), the field pattern is significantly different, as can be seen in Fig. 5. The local field shows extra features that correspond to a higher mode of the tip-surface system. The dipolar character of the fundamental mode makes it fairly efficient in emitting light, as shown by the large scattering cross section associated with it (see Fig. 3(b)). On the contrary, the higher mode has a small scattering cross section associated, which can be explained by the more complex distribution of the local field for this mode.

The formation of such resonances and their energy shifts have significant consequences on the "approach curves", i.e. when varying the tip-surface distance for fixed values of the photon energy. Figure 6 shows the local-field enhancement and the scattering cross section for a photon energy of $1.9 \mathrm{eV}$ and different values of the radius of the sphere. The local-field enhancement increases when the tip approaches the surface for the range of tip-sample distance studied and for all the sphere radii under study [Fig. 6(c)]. (We only show results for tipsurface distances larger than $0.3 \mathrm{~nm}$, since for very short

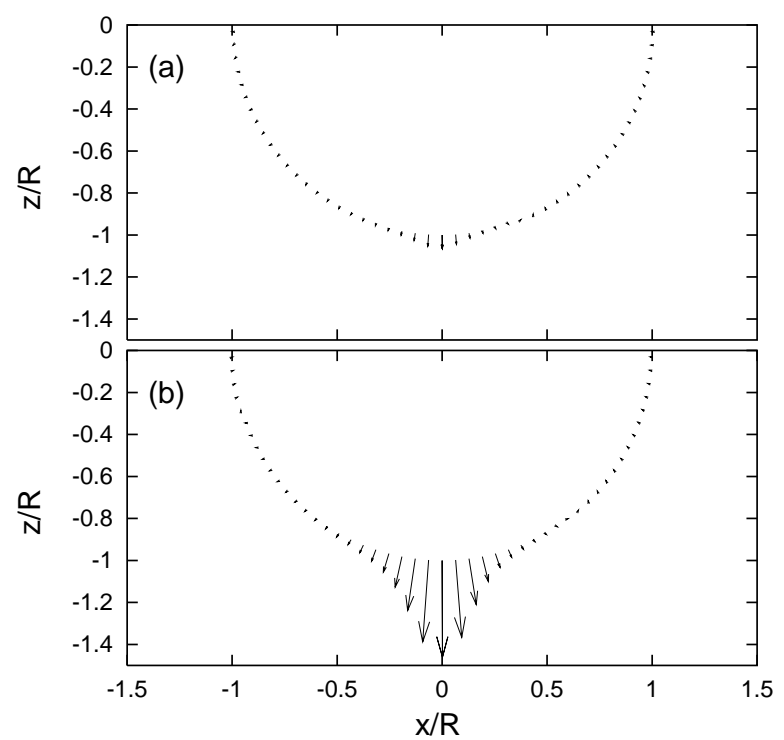

FIG. 4: Real part (a) and imaginary part (b) of the electric field on the sphere surface for $R=80 \mathrm{~nm}, E=1.32 \mathrm{eV}$ and $d=1 \mathrm{~nm}$, which corresponds to the first maximum of the near-field enhancement in Fig. 3(a). The displayed fields have been calculated just outside the surface of the sphere in a vertical plane that contains the incident wave vector $\mathbf{q}$. The absolute magnitude of the depicted fields is about 325 times stronger than the incident field as can be seen in Fig. 3 . 
distances the description of the system with macroscopic dielectric function is no longer justified.33)

Figure 6(a) illustrates the behavior of the differential scattering cross section as a function of tip-surface distance. It presents a maximum. It is worth noticing that this maximum does not imply a maximum of the local field enhancement, which keeps increasing when the sphere approaches the surface. The corresponding feature in the local field enhancement is a shoulder, rather than a maximum, as can be seen in Fig 6 (c) for sphere radius $\mathrm{R}=40 \mathrm{~nm}$. The tip-surface distance at which the maximum of the scattered field appears increases with the radius of the sphere and the peak becomes wider. In addition, some extra structure appears at very short distances [see fig. 6(b)], especially for the larger radii. We will analyze the physical origin of the different features by looking in detail at the local field distribution.

Figure 7 displays the local field around the sphere for the maximum of the scattering cross section for a sphere with radius $40 \mathrm{~nm}$. This figure shows that the peak corresponds to the fundamental surface plasmon mode of the tip-surface system. The approach curves for small spheres were previously calculated by Girard,14 who modeled the sphere by an electric dipole and obtained peaks that can be related to the fundamental surface plasmon mode. For the larger radii under study, the fundamental mode resonance is already shifted to energies lower than $1.9 \mathrm{eV}$, the photon energy considered in Fig. 6. The behavior of the scattering cross section as a function of tip-surface distance is in this case controlled by the tail of the resonance peaks shown in Fig.

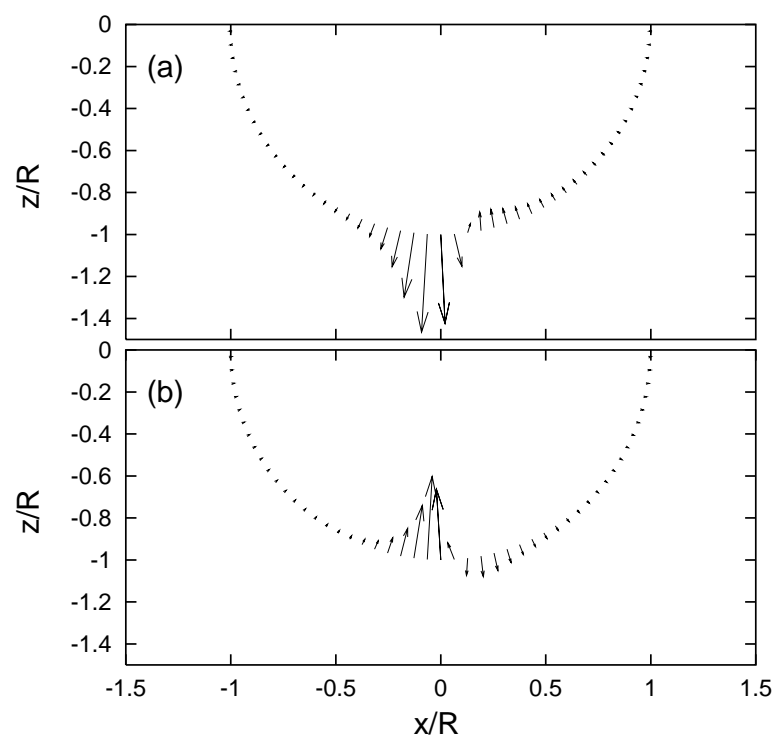

FIG. 5: Real part (a) and imaginary part (b) of the electric field on the sphere surface for $R=80 \mathrm{~nm}, E=1.9 \mathrm{eV}$ and $d=1 \mathrm{~nm}$, which corresponds to the second maximum (with a magnitude $\sim 300$ times that of the incident field) of the near-field enhancement in Fig. B(a).
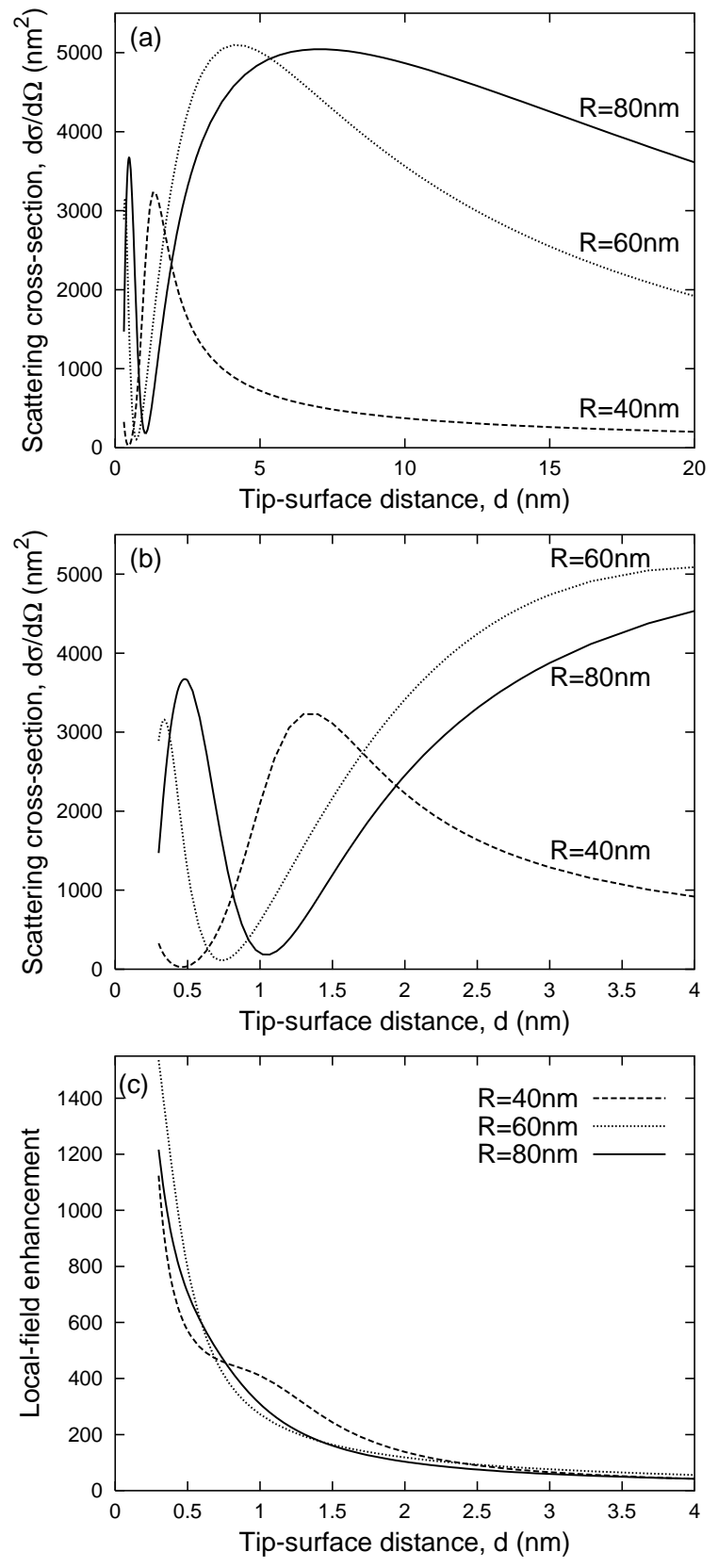

FIG. 6: Differential scattering cross section (a) and (b), and near-field enhancement just underneath the sphere (c), as a function of the tip-surface distance $d$, for a photon energy of $1.9 \mathrm{eV}$ and sphere radius $R$ of $40 \mathrm{~nm}, 60 \mathrm{~nm}$, and $80 \mathrm{~nm}$. The angle of incidence is $\theta_{\text {in }}=57^{\circ}$ and the angles of observation are $\theta_{\text {out }}=57^{\circ}$ and $\phi_{\text {out }}=90^{\circ}$.

3. This explains why the peaks in the scattering cross section as a function of tip-sample distance displayed in Fig. 6(a) and calculated for larger sphere radii $(60 \mathrm{~nm}$ and $80 \mathrm{~nm}$ ) are much wider than the one calculated for the $40-\mathrm{nm}$ sphere. At a much smaller tip-surface distance, i.e. about $0.5 \mathrm{~nm}$, a narrow peak is present for the $R=80 \mathrm{~nm}$ sphere [Fig. 6(b)]. The local field distribution displayed in Fig. 8, with its characteristic node, shows 
that this peak is associated with the higher mode resonance of the tip-surface system. The tip-sample distance at which this higher-mode resonance appears increases with increasing sphere radius and photon energy.

When studying the light scattered by an isolated sphere one finds that for the larger sphere $(R=80 \mathrm{~nm})$ there is a Mie resonance at energies near $1.9 \mathrm{eV}$. When considering the sphere-surface system, the Mie resonance of the isolated sphere implies that, for the $80 \mathrm{~nm}$-radius sphere, the amount of s-polarized scattered light becomes larger than the p-polarized light. Nevertheless, the amount of s-polarized scattered light is almost independent of the tip-sample distance, except for distances shorter than $1 \mathrm{~nm}$, where some features appear that could be related to the formation of the higher resonant mode discussed above.

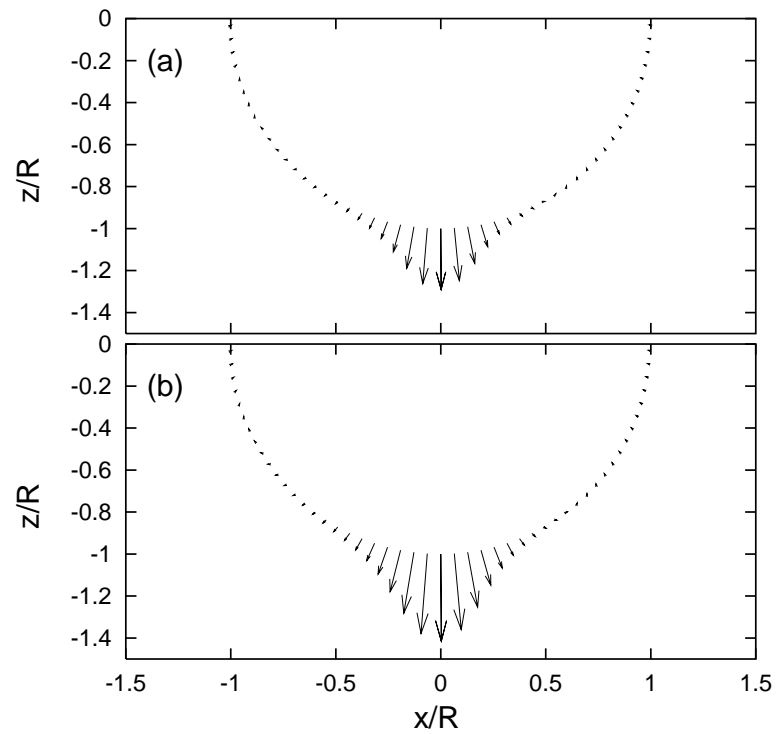

FIG. 7: Real part (a) and imaginary part (b) of the electric field on the sphere surface for $R=40 \mathrm{~nm}, E=1.9 \mathrm{eV}$ and $d=1.23 \mathrm{~nm}$, which corresponds to a peak of scattering cross section in Fig. 6.(b). The local field is about 400 times stronger than the incident field in this case as can be seen in Fig. 6(c).

It is also interesting to analyze the approach curve for photon energies with no significant resonance effects. Figure 9(a) shows the local-field enhancement for a photon energy of $2.4 \mathrm{eV}$. In this "off-resonance" situation, the local-field enhancement presents a maximum as a function of the tip-sample distance. This maximum appears between $\mathrm{d}=1 \mathrm{~nm}$ and $\mathrm{d}=3 \mathrm{~nm}$ for the sphere radii studied. The maximum of the local field enhancement manifests itself also in the scattering cross section through a very small peak at similar tip-surface distance [see Fig. 9(b)]. However, the scattering cross-section maximum appears at much larger tip-surface distances, between $\mathrm{d}=10 \mathrm{~nm}$ and $\mathrm{d}=20 \mathrm{~nm}$.

In order to gain physical insight about the origin of the different maxima in the off-resonance situation

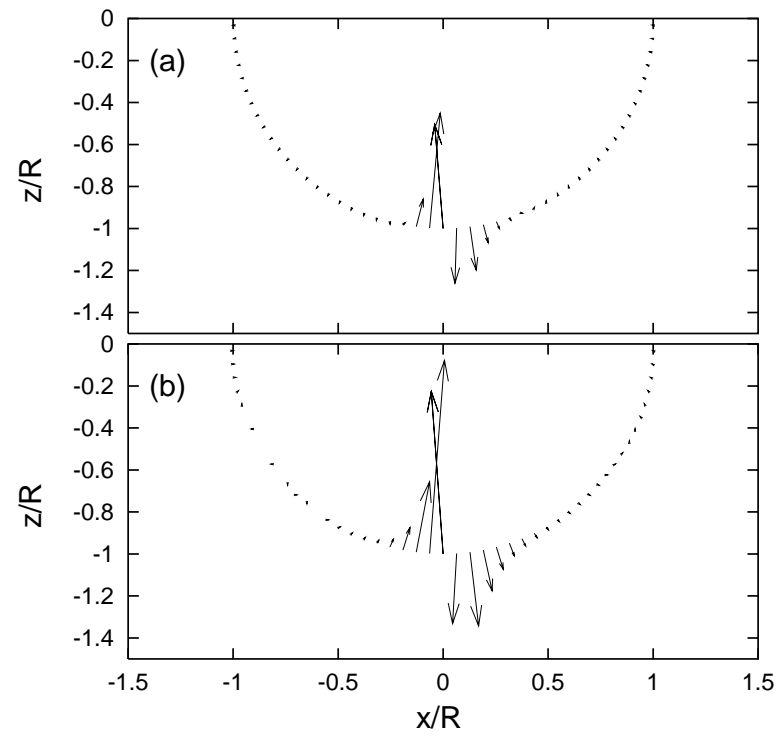

FIG. 8: Real part (a) and imaginary part (b) of the electric field on the sphere surface for $R=80 \mathrm{~nm}, E=1.9 \mathrm{eV}$ and $d=0.48 \mathrm{~nm}$, which corresponds to a peak of scattering cross section in Fig. 6(b). From Fig. 6(c) we see that the local field in this case is about 1000 times stronger than the incident field.

$(E=2.4 \mathrm{eV})$, we study the convergence with the number of multipoles taken into account in the calculation. We analyze the case of a sphere radius of $40 \mathrm{~nm}$, for which the maxima of enhancement and scattered amplitude appear at well separated values of tip-surface distance. The local-field enhancement requires a high number of multipoles to converge, in this particular case it was necessary to consider multipoles of order $l=20$ to arrive at a satisfactory convergence. Differently, convergence for the scattering cross section is achieved with a low number of multipoles. Retaining multipoles up to $l=2$, i.e. dipole and quadrupole modes of the sphere, we obtain most of the features of the scattering cross section, in particular, its main maximum. This fast convergence implies that a simple model for the sphere-surface system can be adequate for the estimation of the scattering off the system when no resonant behavior is involved. In particular, the often used model consisting of a dipole representing the sphere and its image dipole that together have a quadrupole character might be suitable in this situation. If we are interested in the local-field enhancement, even in this off resonance situation, we need to consider a more sophisticated model including higher multipoles of the sphere.

On the other hand, for a photon energy of $1.9 \mathrm{eV}$ (the "on-resonance" situation for which cavity modes can be built up), it was necessary to take into account a high number of multipoles in order to converge both the scattering cross section and the local field enhancement. For example, for a sphere radius of $60 \mathrm{~nm}$, for reproducing the main wide peak of the scattering cross section it was 

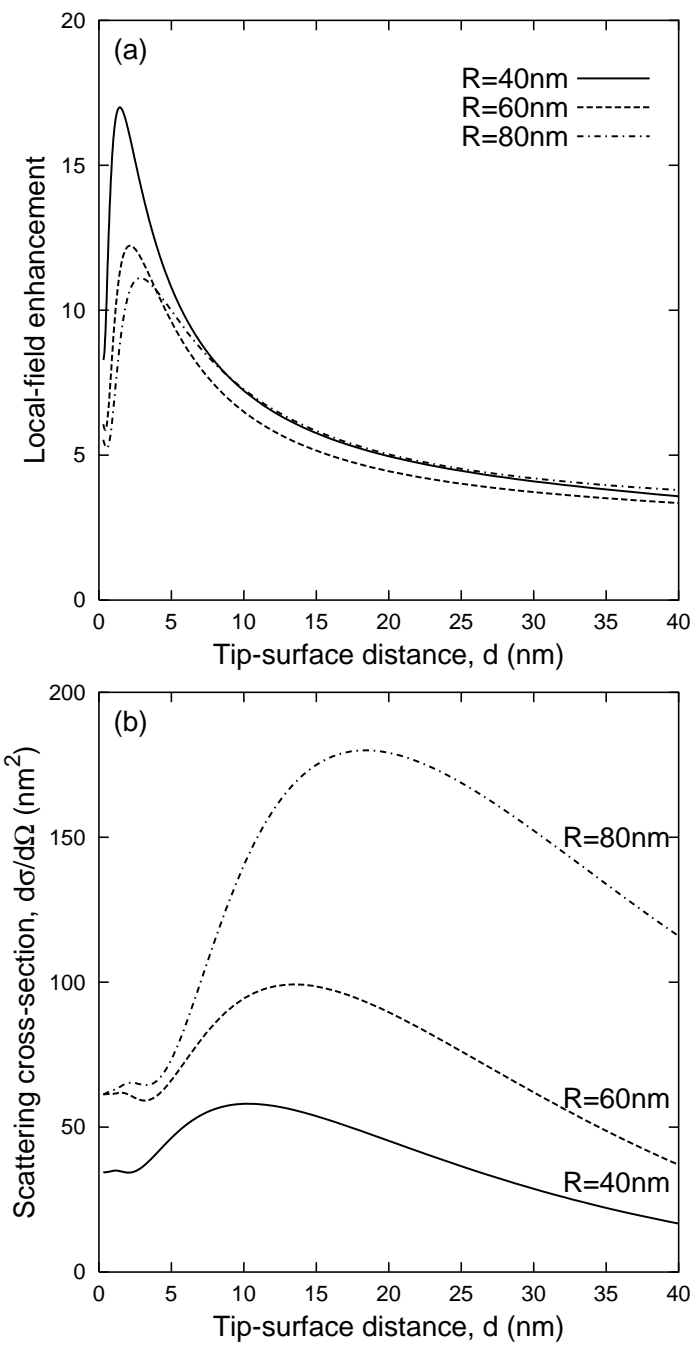

FIG. 9: (a) Near-field enhancement just underneath the sphere, and (b) differential scattering cross section as a function of the tip-surface distance $d$, for a photon energy of $2.4 \mathrm{eV}$ and sphere radii of $40 \mathrm{~nm}, 60 \mathrm{~nm}$, and $80 \mathrm{~nm}$, respectively. The angle of incidence is $\theta_{\text {in }}=57^{\circ}$ and the angles of observation are $\theta_{\text {out }}=57^{\circ}$ and $\phi_{\text {out }}=90^{\circ}$.

enough to retain multipoles up to around $l=10$. However, we needed to take into account multipoles up to the order of $l=70$ to arrive at convergence for the local field enhancement and to reproduce the peak of the scattering cross section at short sphere-surface distance associated with a higher resonant mode. For the range of energies where tip-surface plasmon modes can be built up, a simple model is inadequate to describe the system.

Cavity or gap modes hay been reported in the context of light emission in STM 345 and in samples consisting of metallic nanoparticles placed very close to a metallic surface. 36 In both cases, the distance between the metallic tip or nanoparticle and the metallic surface can be shorter than 1 nanometer. For SNOM, little has been done in connection with gap modes. In the context of apertureless SNOM, the approach curves have beepeyporimentally studied in detail by several groups. A usual effect is the appearance of a peak or maximum of the signal when tip approaches the sample, especially when both the tip and the sample are made of the same metal. 39 This peak, which is sensitive to the polarization and the dielectric functions of the tip and the sample, can be interpreted as a manifestation of the fundamental cavity mode discussed in this article. In a recent article 41 André et al. have reported experimental results on apertureless SNOM with a STM that might be related to the formation of cavity modes between the tip and the surface. Particularly, different behaviors of the signal as a function of the tip-substrate distance have been measured, with the appearance of minima and maxima as the tip is removed from the substrate. There are some qualitative similarities with the results shown in Fig. 6, supporting the claim that the effects observed in Ref. 41 are associated with cavity modes. Nevertheless, the experimental setup in Ref. 41 is different than the one considered in the present work, therefore the comparison should be established with caution.

So far, we have considered sphere-surface distances much shorter than the wavelength. For sphere-surface separations on the order or larger than the wavelength of the incident wave, both the scattering cross-section and the near-field enhancement oscillate as a function of the tip-surface distance. These undulations are due to the interference of the incident wave and the wave reflected by the surface. At this range of sphere-surface distance, there is no significant coupling between the sphere and the sample.

\section{CONCLUSIONS}

By means of a detailed theoretical framework, which includes retardation, higher multipoles of the tip, and multiple scattering between the tip and the sample, we have studied the effects associated with the tip-sample coupling in apertureless SNOM. In particular, we have analyzed the implications of energy shifts of the tipinduced surface plasmons or cavity modes as the tip approaches the surface. There is no exact correspondence between the local field enhancement and the scattering cross section. As a function of the tip-surface distance, the scattering cross section present maxima related to the cavity modes, while the local field enhancement keeps increasing as the tip approaches the surface. For a photon energy for which resonant effects are negligible, both the local field enhancement and the scattering cross section present maxima but at well separated values of the tip-surface distance, in contrast to what is sometimes assumed. In some cases, the maximum of the scattering cross section appears at tip-surface distances of $15 \mathrm{~nm}$ while the maximum local-field enhancement is at a tipsurface distance of approximately $2 \mathrm{~nm}$. 
We have also studied the convergence of the results in terms of the number of multipoles included in the calculation. In general, one must retain a high number of multipoles in order to arrive at convergence. Only for energies for which no cavity modes are built up, a simple model (retaining dipole and quadrupole modes of the sphere) for the sphere-surface system can be appropriate for estimating the light scattered off the system, but insufficient for the local field enhancement. When tipsurface plasmon modes are present, the simple model is inadequate and a high number of multipoles should be taken into account.

Finally, we would like to stress that the present results arealso significant for surface-enhanced Raman scattering 42 and the emerging area of tip-enhanced Raman spec- troscopy 4344.45 The formation of cavity modes between the tip and the sample and the associated field enhancement can play a significant role. 46

\section{Acknowledgments}

JAP gratefully acknowledges financial support from the European Union through TMR network Electromagnetic Interactions in Tunneling (Contract No. ERBFMRX-CT98-0198). The research of PJ and SPA is further supported by the Swedish Natural Science Research Council. The work of PJ is also supported by the SSF through the Nanometer Consortium at Lund University.
1 D. W. Pohl, W. Denk, and M. Lanz, Appl. Phys. Lett. 44, 651 (1984); U. Dürig, D. W. Pohl, and R. Rohner, J. Appl. Phys. 59, 3318 (1986).

2 A. Lewis, M. Isaacson, A. Harootunian, and A. Murray, Ultramicroscopy 13, 227 (1984); A. Harootunian, E. Betzig, M. Isaacson, and A. Lewis, Appl. Phys. Lett. 49, 674 (1986).

3 M. A. Paesler and P. Moyer, Near-Field Optics: Theory, Instrumentation, and Applications (Wiley-Interscience, 1996).

${ }^{4}$ U. Ch. Fischer and D. W. Pohl, Phys. Rev. Lett. 62, 458 (1989).

5 M. Specht, J. D. Pedarnig, W. M. Heckl, and T. W. Hänsch, Phys. Rev. Lett. 68, 476 (1992).

${ }^{6}$ F. Zenhausern, M. P. O'Boyle, and H.K. Wickramasinghe, Appl. Phys. Lett. 65, 1623 (1994); F. Zenhausern, Y. Martin, and H. K. Wickramasinghe, Science 269, 1083 (1995).

7 Y. Inouye and S. Kawata, Opt. Lett. 19, 159 (1994).

8 P. Gleyzes, A. C. Boccara, and R. Bachelot , Ultramicroscopy 57, 318 (1995).

9 E. J. Sánchez, L. Novotny, X. S. Xie, Phys. Rev. Lett. 82, 4014 (1999).

10 B. Knoll and F. Keilmann, Nature (London) 399, 134 (1999).

11 C. Girard and A. Dereux, Rep. Prog. Phys. 59, 657 (1996).

12 J. J. Greffet and R. Carminati, Prog. Surf. Science 56, 133 (1997).

13 J. M. Vigoureux, C. Girard, and D. Courjon, Opt. Lett. 14, 1039 (1989).

14 C. Girard, Appl. Opt. 31, 5380 (1992).

${ }^{15}$ W. Denk and D. W. Pohl, J. Vac. Sci. Technol. B 9, 510 (1991).

16 D. van Labeke and D. Barchiesi, J. Opt. Soc. Am. A 9, 732 (1992); 10, 2193 (1993); D. Barchiesi and D. van Labeke, J. Modern Optics 40, 1239 (1993).

17 A. Madrazo, M. Nieto-Vesperinas, and N. García, Phys. Rev. B 53, 3654 (1996); A. Madrazo, R. Carminati, M. Nieto-Vesperinas, and J.-J. Greffet, J. Opt. Soc. Am. A 15, 109 (1998).

18 O. J. F. Martin and C. Girard, Appl. Phys. Lett. 70, 705 (1997); O. J. F. Martin and M. Paulus, J. Microscopy 205, 147 (2001).

19 L. Novotny, R. X. Bian, and X. S. Xie, Phys. Rev. Lett.
79, 645 (1997).

${ }^{20}$ H. Furukawa and S. Kawata, Opt. Commun. 148, 221 (1998).

21 A. V. Zayats, Opt. Commun. 161, 156 (1999).

${ }^{22}$ L. Aigouy, F. X. Andréani, A. C. Boccara, J. C. Rivoal, J. A. Porto, R. Carminati, J.-J. Greffet, and R. Mégry, Appl. Phys. Lett. 76, 397 (2000).

23 J. Aizpurua, S. P. Apell, and R. Berndt, Phys. Rev. B 62, 2065 (2000).

24 J. A. Porto, R. Carminati, and J.-J. Greffet, J. Appl. Phys. 88, 4845 (2000).

25 J. N. Walford, J. A. Porto, R. Carminati, J.-J. Greffet, P. M. Adam, S. Hudlet, J.-L. Bijeon, A. Stashkevich, and P. Royer, J. Appl. Phys. 89, 5159 (2001).

26 P. Johansson, S. P. Apell, and D. R. Penn, Phys. Rev. B 64, 054411 (2001).

27 J. N. Walford, J. A. Porto, R. Carminati, and J.-J. Greffet, J. Opt. Soc. Am. A 19, 572 (2002).

28 P. K. Aravind and H. Metiu, Surf. Sci. 124, 506 (1983).

${ }^{29}$ H. Metiu, Prog. Surf. Sci. 17, 153 (1984).

30 R. W. Rendell and D. J. Scalapino, Phys. Rev. B, 24, 3276 (1981).

31 J. D. Jackson, Classical Electrodynamics (Wiley, New York, 1999).

32 Handbook of Optical Constants of Solids, edited by E. D. Palik (Academic, Orlando, 1985).

33 For a discussion on the validity of an approach employing local dielectric functions, see P. Johansson, Phys. Rev. B 58, 10823 (1998).

34 R. Berndt, J. K. Gimzewski, and P. Johansson, Phys Rev. Lett. 67, 3796 (1991); 71, 3493 (1993).

35 N. Nilius, N. Ernst, H.-J. Freund, and P. Johansson, Phys. Rev. B 61, 12682 (2000).

36 T. Kume, N. Nakagawa, S. Hayashi, and K. Yamamoto, Solid-State Commun. 93, 171 (1995); T. Kume, S. Hayashi, and K. Yamamoto, Phys. Rev. B 55, 4774 (1997).

37 Y.-K. Kim, P. M. Lundquist, J. A. Helfrich, J. M. Mikrut, G. K. Wong, P. R. Auvil, and J. B. Ketterson, Appl. Phys. Lett. 66, 3407 (1995); A. E. Kryukov, Y.-K. Kim, and J. B. Ketterson, J. Appl. Phys. 82, 5411 (1997).

38 J. Koglin, U. C. Fischer, and H. Fuchs, Phys. Rev. B 55, 7977 (1997).

39 J. Ferber, U. C. Fischer, N. Hagedorn, and H. Fuchs, Appl. 
Phys. A 1999, 581 (1999).

40 R. Hillenbrand and F. Keilmann, Phys. Rev. Lett. 85, 3029 (2000); Appl. Phys. Lett. 80, 25 (2002).

41 P. André, F. Charra, and M. P. Pileni, J. Appl. Phys. 91, 3028 (2002).

42 S. Nie and S. R. Emory, Science 275, 1102 (1997).

${ }^{43}$ R. M. Stöckle, Y. D. Suh, V. Deckert, and R. Zenobi,
Chem. Phys. Lett. 318, 131 (2000).

44 M. S. Anderson, Appl. Phys. Lett. 76, 3130 (2000).

45 N. Hayazawa, Y. Inouye, Z. Sekkat, and S. Kawata, Chem. Phys. Lett. 335, 335 (2001).

46 D. L. Mills, Phys. Rev. B 65, 125419 (2002). 